\title{
A Genetic Algorithm for Solving Knapsack Problems Based on Adaptive Evolution in Dual Population
}

\author{
YAN Tai-shan, GUO Guan-qi, Li Hong-min, He Wei \\ School of Information and Communication Engineering \\ Hunan Institute of Science and Technology \\ Yueyang, Hunan, China \\ Yantaishan163@163.com
}

\begin{abstract}
In order to solve knapsack problems efficiently, an improved genetic algorithm based on adaptive evolution in dual population (called DPAGA) is proposed. In DPAGA, the new population produced by selecting operation is regarded as main population. The population composed by the individuals washed out by selecting operation is regarded as subordinate population. The individual evolution strategy of main population is different from that of subordinate population. The crossover operators and mutation operators are all adjusted non-linearly and adaptively. DPAGA is used to solve knapsack problems. The experimental results show that its convergence speed and solution quality are all better then that of simple genetic algorithm. It is also suited to solve other optimization problems.
\end{abstract}

Keywords- Genetic algorithm; Dual population; Adaptive evalution; Knapsack problem

\section{INTRODUCTION}

Knapsack problem[1-7] is a well-known and well-studied problem in combinatorial optimization. It has a wide range of applications, for example network planning, network routing, parallel scheduling, and budgeting etc.. Mathematically, the 0/1 knapsack problem may be formulated as:

$$
\begin{gathered}
\text { Maximize: } f\left(x_{1}, x_{2}, \cdots, x_{n}\right)=\sum_{i=1}^{n} v_{i} x_{i} \\
\text { Subject to: } \sum_{i=1}^{n} w_{i} x_{i} \leq c
\end{gathered}
$$

where, $x_{i} \in\{0,1\}, i=1,2, \cdots, n$. $C$ denotes the capacity of knapsack, $v_{i}$ denotes the value of object $\mathrm{i}$, and $w_{i}$ denotes the weight of object i. Generally, we assume that $w_{i}, v_{i}$ and $C$ are all positive integers.

Since the knapsack problem is a NP problem, the traditioanal algorithms have a large calculation and need a long running time when solve the knapsack problems with large scale[8]. In order to solve knapsack problems efficiently, an improved genetic algorithm based on adaptive evolution in dual population will be proposed in this paper.

\section{Genetic Algorithm Based on AdAPTIVE EVOLUTION IN DUAL PopUlation（DPAGA）}

\section{A. Basic Idea of the algorithm}

Genetic algorithm based on adaptive evolution in dual population ( DPAGA) is a novel improved adaptive genetic algorithm. Its population includes a new population formed by selecting operation (the main population) and a population eliminated by selecting operation (the subordinate population). Dual crossover operator and dual mutation operator are introduced in this algorithm. Dual crossover operator means the main crossover operator $P_{b c}$ and the subordinate crossover operator $P_{s c}$. Dual mutation operator means the main mutation operator $P_{b m}$ and the subordinate mutation operator $P_{s m}$. In the evolution of the main population, the individuals perform adaptive crossover operation with high probability and adaptive mutation operation with low probability according to the main crossover operator and the main mutation operator. And in the evolution of the subordinate population, the individuals perform adaptive crossover operation with low probability and adaptive mutation operation with high probability according to the subordinate crossover operator and the subordinate mutation operator.

\section{B. Key operators}

In the genetic algorithm based on adaptive evolution in dual population, key operators include selection operator, dual crossover operator and dual mutation operator. Selection operator is often realized by the roulette wheel selection method. The dual crossover operator and dual mutation operator are adjusted adaptively according to the individual fitness in the evolution of population. Their formulas can be described as follows:

$P_{b c}=\left\{\begin{array}{cl}P_{b c \min }+\frac{P_{b c \max }-P_{b c \min }}{1+\exp \left(-\left(\frac{f^{\prime}-f_{b a v g}}{f_{b \max }-f_{b a v g}}\right)\right)}, & f^{\prime} \geq f_{\text {bavg }} \\ P_{b c \max }, & f^{\prime}<f_{\text {bavg }}\end{array}\right.$ 


$$
\begin{aligned}
& P_{b m}=\left\{\begin{array}{cl}
P_{b m \text { min }}+\frac{P_{b m \text { max }}-P_{b m \text { min }}}{1+\exp \left(-\left(\frac{f-f_{\text {bavg }}}{f_{b \max }-f_{\text {bavg }}}\right)\right)}, & f \geq f_{\text {bavg }} \\
P_{b m \text { max }}, & f<f_{\text {bavg }}
\end{array}\right. \\
& P_{s c}=\left\{\begin{array}{cc}
P_{s c \min }+\frac{P_{s c \max }-P_{\text {sc min }}}{1+\exp \left(-\left(\frac{f^{\prime}-f_{\text {savg }}}{f_{\text {s } \max }-f_{\text {savg }}}\right)\right)}, & f^{\prime} \geq f_{\text {savg }} \\
P_{\text {sc } \max }, & f^{\prime}<f_{\text {savg }}
\end{array}\right. \\
& P_{s m}=\left\{\begin{array}{cl}
P_{s m \text { min }}+\frac{P_{s m \text { max }}-P_{s m \text { min }}}{1+\exp \left(-\left(\frac{f-f_{\text {savg }}}{f_{s \max }-f_{\text {savg }}}\right)\right)}, & f \geq f_{\text {savg }} \\
P_{s m \text { max }}, & f<f_{\text {savg }}
\end{array}\right.
\end{aligned}
$$

Where, $f_{b \max }$ and $f_{s \max }$ denotes the maximal fitness of the main population and subordinate population respectively, $f_{\text {bavg }}$ and $f_{\text {savg }}$ denotes the average fitness of the main population and subordinate population respectively, $f^{\prime}$ denotes the higher fitness of the two crossing individuals, $f$ denotes the fitness of the mutating individuals, $P_{b c \text { min }}$ and $P_{b c \text { max }}$ denotes the lower limit and the upper limit of the crossover probability of the main population respectively, $P_{s c \text { min }}$ and $P_{s c \max }$ denotes the lower limit and the upper limit of the crossover probability of the subordinate population respectively, $P_{b m \text { min }}$ and $P_{b m \text { max }}$ denotes the lower limit and the upper limit of the mutation probability of the main population respectively, $P_{s m \text { min }}$ and $P_{s m \text { max }}$ denotes the lower limit and the upper limit of the mutation probability of the subordinate population respectively. Generally, their values may chosen as follows. $P_{b c \min }=0.6, P_{b c \max }=0.9$,

$$
\begin{aligned}
& P_{s c \text { min }}=0.001, \quad P_{s c \text { max }}=0.1, \quad P_{b m \text { min }}=0.001, \\
& P_{b m \text { max }}=0.1, \quad P_{s m \text { min }}=0.1, \quad P_{s m \text { max }}=0.5 .
\end{aligned}
$$

From formula (1) formula (4), we can see that the probability of crossover and the probability of mutation are adjusted nonlinearly according to the function $f(x)=\frac{1}{1+\exp (-x)}$ between the average fitness and the maximal fitness. When the fitness of the majority of individuals is near and the average fitness is close to the maximal fitness, the probability of crossover and the probability of mutation will be elevated. So, the individuals nearby the maximal fitness are preserved as many as possible. The ability of the algorithm to jump out the local convergence is enhanced greatly.

\section{Steps and working flow of the algorithm}

The working flow of DPAGA can be described as follows:

Step1: Code genetic individuals and initialize population $P_{t}$;

Step2: Calculate individual fitness;

Step3: Perform selecting operation according to the roulette wheel selection method, and gain the new population $P_{t+1}$ (main population) and the eliminated population $P_{t+1}^{\prime}$ (subordinate population);

Step4: For the main population $P_{t+1}$, perform adaptive crossover operation with high probability and adaptive mutation operation with low probability;

Step5: Judge convergence condition of $P_{t+1}$. If it is congruous, perform Step9;

Step6: For the subordinate population $P_{t+1}^{\prime}$, perform adaptive crossover operation with low probability and adaptive mutation operation with high probability;

Step7: Form the new population $P_{t}$ from the results of Step4 and Step6;

Step8: Repeat Step2 Step6, till the convergence condition is congruous;

Step9: Stop.

Fig.1 illustrates the working flow of DPAGA.



Figure 1. The working flow of DPAGA 


\section{EXPERIMENTS ON KNAPSACK PROBLEMS}

In order to test the performance and effectiveness of the algorithm, the following two examples of knapsack problem are chosen in the experiment.

Example 1 The knapsack problem composed of 50 objects. It is described as:

$V=220,208,198,192,185,180,165,162,160,158$, $155,130,125,122,120,118,115,110,105,101,100,100$, 98, 96, 95, 90, 88, 82, 80, 77, 75, 73, 72, 70, 69, 66, 65, 63, $60,58,56,50,30,25,15,10,8,5,3,1\}$,

$W=\{80,82,85,70,72,70,66,50,55,25,50,55,40$, $48,50,32,22,60,30,32,40,38,35,32,25,28,30,22,50$, $30,45,30,60,50,20,65,20,25,30,10,20,25,15,10,10$, $10,4,4,2,1\}, C=1000$.

Example 2 The knapsack problem composed of 100 objects. It is described as:

$V=\{998,997,991,986,978,977,939,936,924,920$, 911, 901, 901, 885, 880, 866, 866,863, 856, 842, 809, 794, 792, 789, 778, 767, 764, 764, 763, 759,756, 747, 739, 708, 707, 706, 694, 693, 684, 680, 676, 652, 644,640, 628, 12, $607,597,593,570,560,556,556,556,542,538,530,530$, 520, 498, 487, 466, 464, 461, 459, 456, 452, 443, 412,399, 391, 383, 381, 378, 377, 359, 353, 351, 327, 317, 311, 295,289, 287, 283, 269, 249, 248, 235, 193, 189, 189, 134, $108,93,74,51,48,23,8\}$,

$W=\{353,180,377,230,87,174,157,390,186,213$, 56, 86, 77, 215, 252, 90, 360, 187, 294, 299,372, 384, 93, 328, 283, 99, 114, 374, 383, 183, 248, 164, 323, 263,266, $318,296,196,10,324,128,376,19,280,229,225,217$, $134,233,35,361,302,166,374,392,319,241,15,384,82$, $158,322,139,239,110,44,115,23,267,82,30,198,173$, 70, 329, 125, 220,107, 148, 159, 351, 56, 17, 99, 308, 396, 327, 235, 213, 223, 372,376, 191, 299, 304, 277, 292, 391, $120,37\}, \quad C=9803$.

In this study, the simple genetic algorithm (SGA) and DPAGA are used to solve the above two knapsack problems. The parameters are set as: $T=300, M=100, p_{c}=0.7$, $p_{m}=0.1$. The results of example 1 are shown as Table 1 . The results of example 2 are shown as Table 2 .

From Table 1 and Table 2, we can see that when solve the knapsack problems, DPAGA is superior to SGA whether look from convergence speed or the quality of solutions.

\section{CONCLUSION}

In this study, an improved genetic algorithm based on adaptive evolution in dual population was proposed. Its population includes a main populationand a subordinate population. In the main population and subordinate population, the genetic operators are adjusted adaptively and nonlinearly with different strategies. The premature convergence of genetic algorithm is conquered and its global searching ability is enhanced greatly. Successful results were gained when experiments were taken on knapsack problems. Its convergence speed and solution quality are all better then that of simple genetic algorithm. It is also suited to solve other optimization problems.

TABLE I. THE OPTIMAL SOLUTION OF EXAMPLE 1

\begin{tabular}{|c|c|c|c|c|}
\hline Algorithm & Code of the optimal solution & $\begin{array}{c}\text { Total } \\
\text { value }\end{array}$ & $\begin{array}{c}\text { Total } \\
\text { weight }\end{array}$ & Time \\
\hline SGA & $\begin{array}{c}1101101111101001101111111 \\
1010000001010011000000011\end{array}$ & 3082 & 999 & 0.432 \\
\hline DPAGA & $\begin{array}{l}1001110111101101101101111 \\
1110100001010011001001000\end{array}$ & 3105 & 1000 & 0.229 \\
\hline
\end{tabular}

TABLE II. THE OPTIMAL SOLUTION OF EXAMPLE 2

\begin{tabular}{|c|c|c|c|c|}
\hline Algorithm & Code of the optimal solution & $\begin{array}{c}\text { Total } \\
\text { value }\end{array}$ & $\begin{array}{c}\text { Total } \\
\text { weight }\end{array}$ & Time \\
\hline SGA & $\begin{array}{l}111111111111111111000111 \\
1100111111001111011111111 \\
0010000101101011110110010 \\
1010001110000000000000000\end{array}$ & 40599 & 9796 & 6.152 \\
\hline DPAGA & $\begin{array}{l}111111111111111110100111 \\
1100111111101101011111111 \\
0010000101101011110110010 \\
1010001110000000000000000\end{array}$ & 40611 & 9799 & 3.627 \\
\hline
\end{tabular}

\section{ACKNOWLEDGMENTS}

Support From the Science Research Project Foundation of Hunan Province Education Department under Grant No. 10C0757, the National Natural Science Foundation of China under Grant No. 60975049, the Natural Science Foundation of Hunan Province under Grant No.11JJ2037 is gratefully acknowledged.

\section{REFERENCES}

[1] Lee Hae Sang, Lie Chang Hoon. A computation method for evaluating importance-measures of Gates in a fault tree. I EEE Trans. on Reliability , 1997 , 46 (3) :360 - 365

[2] WANG Li, SHAO Ding-hong, LU Jin-gui. The Genetic Algorithm of Solving 0/1knapsack Problem. Computer Simulation, 1998,23(3):154 156

[3] LI Kenli, LI Qinghua. An Adaptive algorithm for knapsack problem.Computer research and development,2004,7(41):1292-1297

[4] ZHU Xiaorong, ZHANG Xinghua. A quantum genetic algorithm with repair function and its application in knapsack question. Computer pplications,2007,22(5):1187-1190

[5] HUANG Xiyue, ZHANG Zhuhong.Morden intelligent algorithm theory and application.Beijing: Science Publishing Company,2005.

[6] He Yichao, Liu Jianqin etc..A GREEDY DS_BPSO ALGORITHM FOR GENERAL KNAPSACK PROBLEM. Computer Applications and Software,2008,25(4):230-232

[7] YAN Tai-shan, He Wei. An Algorithm For Solving Knapsack Problems Utilizing Knowledge Evolution Principle. 2011 3nd International IEEE Workshop on Intelligent Systems and Applications (ISA2011), Wuhan, China, May 28-29, 2011, Vol.1: 1-4

[8] Li, K.L., G.M. Dai and Q.H. Li. A genetic algorithm for the unbounded knapsack problem. Proceedings of the 2nd International Conference on Machine Learning and Cybernetics, USA., 2003, 1586-1590. 